\title{
LABA-Einsatz bei Asthma völlig unbedenklich
}

\author{
Bei mäßig- bis schwergradigem Asthma zeigt der LABA Salmeterol in Fixkombination mit einem \\ Kortikoid weder bei Erwachsenen noch bei Kindern und Jugendlichen unerwünschte Nebenwirkungen.
}

_Kurzwirksame $\beta$-Agonisten (SABA)
werden aufgrund von Berichten über To-
desfälle nicht für die Dauer-, sondern
nur für die Bedarfstherapie bei akutem
Asthma bronchiale empfohlen. Auch die
langwirksamen $\beta$-Agonisten (LABA)
standen zunächst unter dem Verdacht,
die Asthmamortalität zu erhöhen. Die
US-Zulassungsbehörde FDA forderte
von den Herstellern prospektive Studien,
um das Risiko von schweren Asthmaan-
fällen mit Hospitalisierung, Intubatio-
nen und Todesfolge zu ermitteln, wenn
Patienten zu einem topischen Glukokor-
tikoid (ICS) ein LABA erhalten.
Die Firma GSK legte nun eine multi-
zentrische, randomisierte, doppelblinde
Studie mit 11.679 Patienten im Alter ab
12 Jahren mit mäßig- bis schwergradi-
gem Asthma vor, die im Jahr zuvor einen
schweren Anfall gehabt hatten. Sie er- hielten über 26 Wochen das ICS Fluticason entweder allein oder in Kombination mit dem LABA Salmeterol. Primärer Endpunkt war das erste Auftreten eines schweren Asthmaanfalls mit Hospitalisierung, Intubation oder Tod.

67 Patienten mit insgesamt 74 Ereignissen erfüllten die Endpunktkriterien, davon 34 mit 36 Anfällen aus der Kombinationsgruppe und 33 mit 38 Anfällen aus der ICS-Gruppe. Asthmatodesfälle wurden nicht berichtet, zwei Patienten mussten intubiert werden. Für keine der beiden Therapien ergab sich eine Überlegenheit. Das Risiko für schwere Exazerbationen ohne Hospitalisierung lag in der Kombinationsgruppe um $21 \%$ niedriger $(8,2$ vs. $10,2 \%, \mathrm{p}<0,001)$. Diese Patienten benötigten eine kurzfristige systemische und/oder eine Rescue-Therapie mit einem SABA-Präparat.
- Stempel DA, Raphiou ICH, Kral KM et al. Serious asthma events with fluticasone plus salmeterol versus Fluticason alone. NEng/ J Med. 2016;374:1822-9

\section{KOMMENTAR}

Die Daten zeigen erstmals deutlich, dass der LABA Salmeterol kein Risiko für Asthmaexazerbationen oder gar Todesfälle darstellt. Das Kombinationspräparat senkte sogar die Zahl der Asthmaanfälle. Eine parallele, gleich aufgebauten Studie an 6.208 Kindern von 4-11 Jahren kam zum selben Ergebnis [Stempel DA et al. N Engl J Med. 2016;375:840-9]. An Schwächen diskutieren die Autoren die kurze Studiendauer sowie den Ausschluss von Patienten mit instabilem oder lebensbedrohlichem Asthma. Für diese Gruppe gelten die Daten nicht ohne Weiteres. Auch Lungenfunktionsdaten oder Entzündungsparameter zur Abschätzung der Wirksamkeit der Mittel enthalten die beiden Studien nicht.

Prof. Dr. med. Dr. h. c. D. Reinhardt

\section{Wissen Sie, was ein Kerion ist?}

Ein 13-jähriges Mädchen hatte eine langsam wachsende, schmerzhafte Schwellung der Kopfhaut des Hinterkopfs, die sich im Laufe von drei Wochen entwickelt hatte. Wegen des Verdachts auf eine bakterielle
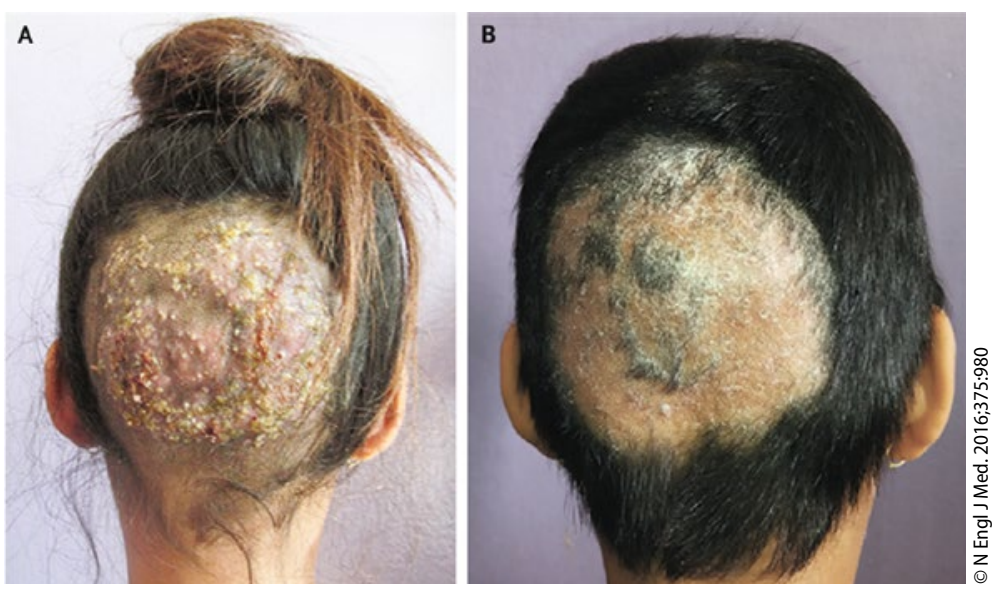

A: Schwellung am Hinterkopf mit Pusteln, serös-blutigem Exsudat und Alopezie. B: Zustand nach sechs Wochen.
Follikulitis hatte sie mehrere Behandlungen mit oralen Antibiotika hinter sich, ohne dass sich der Befund gebessert hätte. Bei der ersten Vorstellung zeigten sich eine gerötete, leicht schmerzhafte Schwellung am Hinterkopf mit pustulösen Veränderungen und einem serös-blutigen Exsudat sowie eine vollständige Alopezie (Abb. A). Ein Schabepräparat der betroffenen Stelle und eine Präparation in 10\%iger Kaliumhydroxidlauge brachte Pilzhyphen zum Vorschein und bestätigte die Diagnose eines Kerions.

Ein Kerion ist eine entzündliche Pilzinfektion der Kopfhaut, die man üblicherweise bei Kindern sieht. Da sie exsudativ und schmerzhaft ist und auch mit einer regionären Lymphadenopathie einhergehen kann, wird sie oft mit einer bakteriellen Follikulitis verwechselt. Die häufigsten Erreger sind Trichophyton verrucosum und Trichophyton mentagrophytes. Meistens verwendet man zur Therapie orales Griseofulvin, obgleich auch andere Antimykotika effektiv sind. Eine Verzögerung von Diagnose und Behandlung führt zu einer narbigen Alopezie. Die Patientin wurde über sechs Wochen hinweg mit Griseofulvin behandelt, worauf sich der Befund deutlich besserte (Abb. B). Die narbige Alopezie wird aber zumindest teilweise persistieren.

Prof. Dr. med. H. S. FüeßI

- Uprety S, Sharma R (maitreeye@gmail.com). Kerion - a boggy lump. N Engl J Med. 2016;375:980 